Provided for non-commercial research and education use. Not for reproduction, distribution or commercial use.

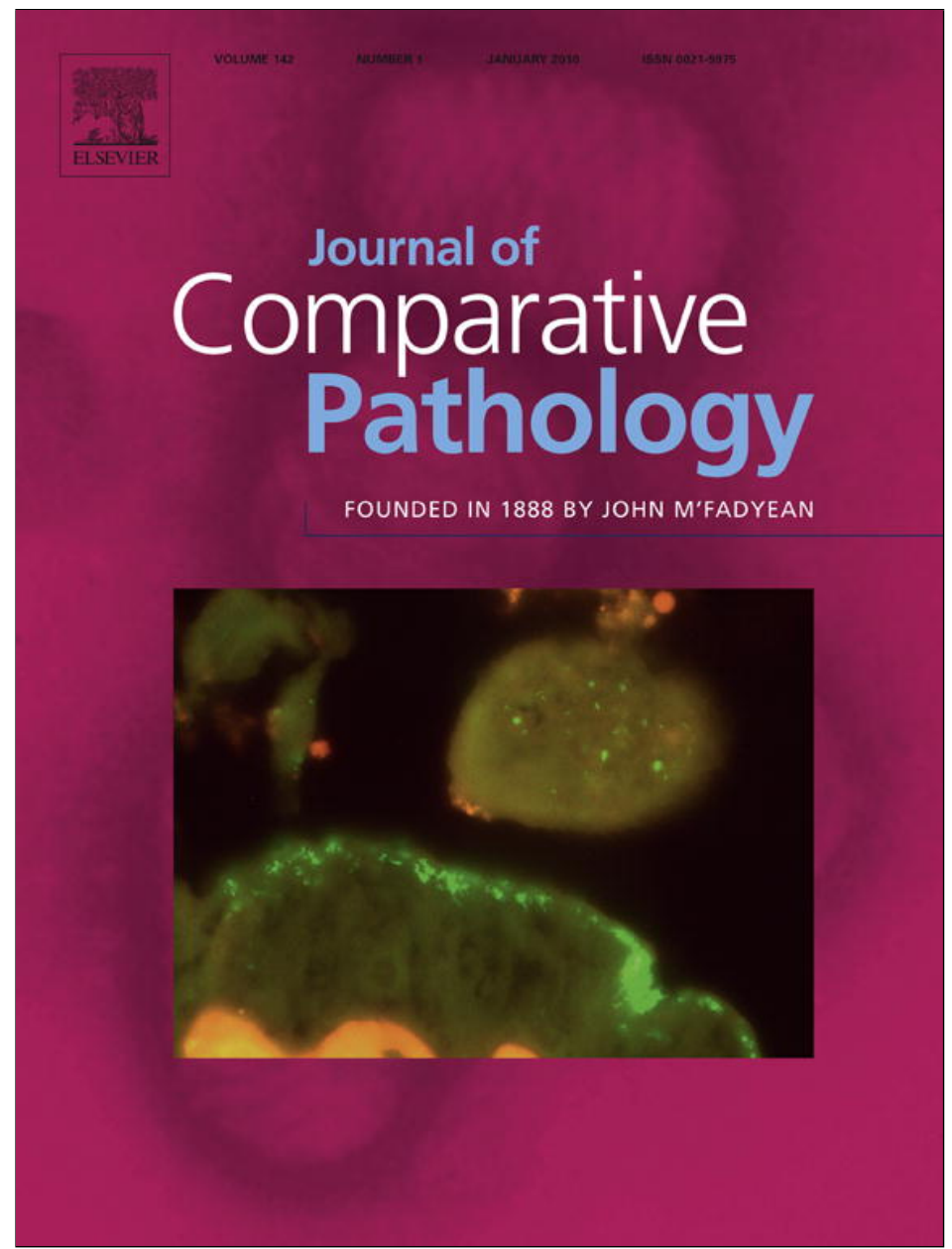

This article appeared in a journal published by Elsevier. The attached copy is furnished to the author for internal non-commercial research and education use, including for instruction at the authors institution and sharing with colleagues.

Other uses, including reproduction and distribution, or selling or licensing copies, or posting to personal, institutional or third party websites are prohibited.

In most cases authors are permitted to post their version of the article (e.g. in Word or Tex form) to their personal website or institutional repository. Authors requiring further information regarding Elsevier's archiving and manuscript policies are encouraged to visit:

http://www.elsevier.com/copyright 


\title{
Immunohistochemical Detection of Aetiological Agents of Proliferative and Necrotizing Pneumonia in Italian Pigs
}

\author{
F. Morandi ${ }^{*}$, F. Ostanello ${ }^{*}$, L. Fusaro ${ }^{*}$, B. Bacci ${ }^{*}$, A. Nigrelli ${ }^{\dagger}$, \\ L. Alborali ${ }^{\ddagger}$, M. Dottori $^{\S}$, F. Vezzoli ${ }^{\|}$, G. Barigazzi $^{\uparrow}$, L. Fiorentini ${ }^{\#}$, \\ V. Sala ${ }^{* *}$, G. Leotti ${ }^{\dagger \dagger}$, F. Joise ${ }^{\dagger \dagger}$ and G. Sarli* \\ * Department of Veterinary Public Health and Animal Pathology, School of Veterinary Medicine, Bologna University, ${ }^{\dagger}$ Istituto \\ Zooprofilattico Sperimentale della Lombardia e dell'Emilia-Romagna (IZSLER), Section of Mantua, ${ }^{\star}$ IZSLER, Section of \\ Brescia, ${ }^{\S} I Z S L E R$, Section of Reggio Emilia, "IZSLER, Section of Lodi, Milan, "IZSLER, Section of Parma, ${ }^{\#}$ IZSLER, \\ Section of Forli, ** Department of Veterinary Public Health and Animal Pathology, School of Veterinary Medicine, Milan \\ University, ${ }^{\dagger \dagger}$ Merial Italia, Milan, Italy and ${ }^{\star \star}$ Merial S.A.S. Lyon, France
}

\begin{abstract}
Summary
Proliferative and necrotizing pneumonia (PNP) is a form of interstitial pneumonia that occurs in weaning and post-weaning pigs. PNP is characterized by hypertrophy and hyperplasia of type II pneumocytes and coagulative necrosis and granular debris within alveolar spaces. Canadian and European studies suggest that the porcine reproductive and respiratory syndrome virus (PRRSV) and porcine circovirus type 2 (PCV2) are the main causes of the disease, but Aujezsky's disease virus (ADV) and swine influenza virus (SIV) have also been considered as potential aetiological agents. An immunohistochemical study was carried out on the lungs of 28 Italian pigs with PNP in order to evaluate the role of PRRSV, PGV2 and ADV in PNP lesions. PRRSV infection was identified in the lungs of 11 pigs, PCV2 in the lungs of four pigs and coinfection with both viruses in the lungs of eight pigs. Neither virus was detected in the lungs of the remaining five pigs. ADV antigen was not detected in any sample. The principle aetiological agent of PNP in Italy therefore appears to be PRRSV. Coinfection with PRRSV and PCV2 is characterized by more severe microscopical changes in affected lungs.
\end{abstract}

(c) 2009 Published by Elsevier Ltd.

Keywords: immunohistochemistry; PCV2; pig; PNP; PRRSV; virus

Proliferative and necrotizing pneumonia (PNP) was first described in Canada in 1990 (Morin et al., $1990)$ in 4-16-week-old post-weaning pigs. PNP is one of the group of "porcine circovirus diseases" (PCVDs) that also includes porcine dermatitis and nephropathy syndrome (PDNS), porcine respiratory disease complex (PRDG), necrotizing lymphadenitis, granulomatous enteritis and reproductive disorders (Harding, 2004; Segalés et al., 2004). On gross necropsy examination, PNP is characterized by consolidation and red-brown to grey discolouration of the

Correspondence to: G. Sarli (e-mail: giuseppe.sarli@unibo.it).

0021-9975/\$ - see front matter doi:10.1016/j.jcpa.2009.06.003 lungs, with lesions generally localized to the cranial and middle lobes and the cranioventral portions of the caudal lobes (Morin et al., 1990). Microscopical examination reveals aggregation of necrotic cells and granular debris within the alveolar spaces together with hyperplasia and hypertrophy of type II pneumocytes (Morin et al., 1990; Larochelle et al., 1994; Drolet et al., 2003; Segalés et al., 2004). Additionally, there is interstitial pneumonia of varying severity, with or without necrotizing bronchiolitis and/ or bronchopneumonia.

The aetiology of PNP remains controversial. The first agent considered in the Canadian studies (Morin 
et al., 1990; Austin and Bystom, 1991; Dea et al., 1992; Girard et al., 1992) was the swine influenza virus (SIV); most recently, Aujeszky's disease virus (ADV) has been implicated (Segalés et al., 1997; Grau-Roma and Segalés, 2007). Larochelle et al. (1994) attributed PNP to porcine reproductive and respiratory syndrome virus (PRRSV), demonstrating the presence of the virus in 28 out of 38 pneumonic lungs. Subsequent Canadian (Drolet et al., 2003) and European studies (Pesch et al., 2000; Grau-Roma and Segalés, 2007) have reported the frequent concurrence of PRRSV and porcine circovirus type 2 (PGV2) in lungs with PNP. The Canadian study commonly demonstrated PRRSV infection in PNP, with concurrent PRRSV and PCV2 in $42 \%$ of cases; however, PRRSV was never the sole aetiological agent recovered. The European study (Grau-Roma and Segalés, 2007) reported PGV2 monoinfection in 29 out of 74 pigs with PNP; PGV2 was associated with PRRSV in 30 animals and PRRSV infection alone occurred in three animals. Both studies failed to detect any aetiological agent in a percentage of lungs with PNP (7 and 10\%, respectively). These results suggest a different aetiopathogenesis for PNP between the two continents.

In addition to PRRSV and PCV2, SIV (Pesch et al., 2000; Drolet et al., 2003; Grau-Roma and Segalés, 2007) and ADV (Grau-Roma and Segalés, 2007) are suspected to be aetiological agents for PNP. ADV is considered to be a causal agent of necrotizing pulmonary lesions in pigs in Spain (Segalés et al., 1997; Grau-Roma and Segalés, 2007), although in those studies only one of 74 pigs with PNP had ADV identified in pulmonary tissue and that animal was coinfected with PGV2 (Grau-Roma and Segalés, 2007). A further three pigs with PNP proved to be coinfected with SIV.

To aim of the present study was to determine the role of PRRSV, PGV2 and ADV in the pathogenesis of PNP in Italian pigs. The study involved immunohistochemical assessment of samples of lung from 28 pigs with PNP. These affected animals were part of an investigation of porcine post-weaning multisystemic wasting syndrome (PMWS) on farms in northern and central Italy (Sarli et al., 2009). Only one of these pigs was SIV seropositive (unpublished data).

Haematoxylin and eosin (HE)-stained sections of lung from 262 pigs were reviewed and samples from 28 animals with histological evidence of PNP were selected for inclusion in the study. Serial sections $(4 \mu \mathrm{m})$ for immunohistochemistry (IHG) were prepared from blocks of paraffin wax-embedded formalin-fixed tissue. Immunohistochemical labelling for PCV2 antigen was performed as described by Sarli et al. (2009). Labelling for PRRSV antigen was performed by application of specific murine monoclonal antibody (clone SDOW 17-A, IgG1 isotype; Rural
Technology Inc., Brookings, South Dakota) and labelling for $\mathrm{ADV}$ was also by murine monoclonal antibody (clone 2H6, IgG1 isotype; Grieco et al., 1997). Sections were initially dewaxed in toluene and rehydrated, and then endogenous peroxidase was blocked with $\mathrm{H}_{2} \mathrm{O}_{2} 3 \%$ in distilled water (for PRRSV) or methanol (for ADV) for $30 \mathrm{~min}$ at room temperature. For ADV labelling, sections were further incubated with protease XIV (0.05\%) for $3 \mathrm{~min}$ at room temperature for antigen retrieval. Sections were then subjected to pre-incubation with "protein block serum-free" (DAKO X0909; Dako, Glostrup, Denmark) for $10 \mathrm{~min}$ at room temperature. Incubation with primary antibody to PRRSV was overnight at $4^{\circ} \mathrm{C}$ and with antibody to ADV for $1 \mathrm{~h}$ at $37^{\circ} \mathrm{C}$. Both primary antibodies were diluted 1 in 50 in Tris buffered saline (TBS, 0.15 M). After washing in TBS, the secondary antibody was applied with subsequent amplification by a commercial streptavidin-biotin-peroxidase technique (DAKO LSAB Kit peroxidase, Dako). Labelling was "visualized" with diaminobenzidine $(0.04 \%$ for $10 \mathrm{~min})$ as chromogen. Sections were counterstained with Papanicolau's haematoxylin, dehydrated and mounted under dibutylphthalate-xylene (DPX). Tissue from an animal known to contain PRRSV (as determined by polymerase chain reaction; PCR) and from the brain of a dog that had died from pseudorabies was used as positive control. Monoclonal antibody of irrelevant specificity (mouse anti-IgG1, DAKO X0931; Dako) was substituted for primary antibody as a negative control.

The lungs from all 28 pigs selected had microscopical evidence of focal to diffuse interstitial pneumonia, with macrophages, lymphocytes and plasma cells infiltrating the alveolar septa. Additionally, there was hypertrophy and hyperplasia of type II pneumocytes and the presence of necrotic cells, occasionally accompanied by basophilic granular debris, within alveolar spaces (Fig.1). Focal necrotizing bronchiolitis was present in four lungs, while 14 lungs had complicating bronchopneumonia. Twenty-two out of the 28 cases $(78.6 \%)$ with PNP were pigs from farms confirmed as affected by PMWS (Sarli et al., 2009), whereas six were derived from farms unaffected by PMWS.

PCV2 antigen was demonstrated in the lungs of 12 animals $(42.8 \%$ of all cases and $54.5 \%$ of animals from farms with confirmed PMWS). In these sections there was strong labelling of the cytoplasm of macrophages in alveolar septa and alveolar spaces (Fig. 2a), and of dendritic cells within bronchial-associated lymphoid tissue (BALT). There was occasional labelling of the necrotic granular debris within alveolar spaces and in one case some hypertrophic pneumocytes also contained PGV2 antigen. In the four lungs 


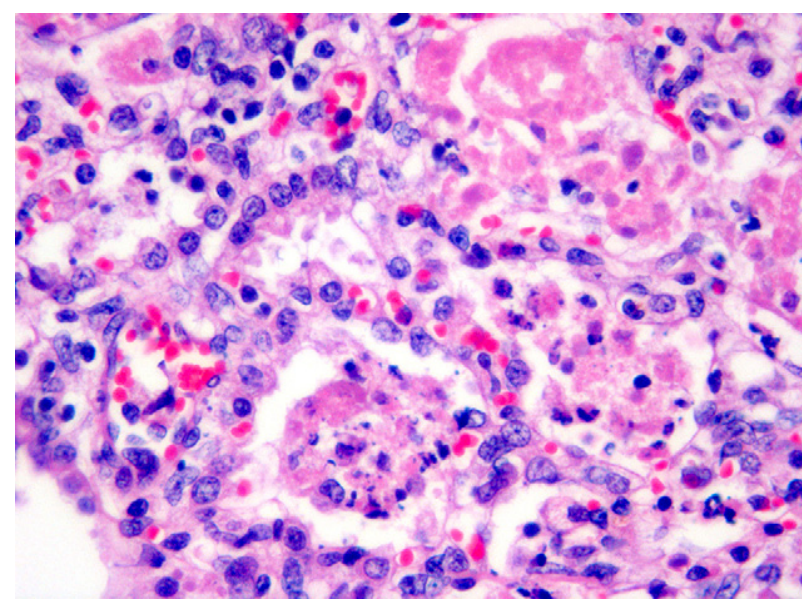

Fig. 1. Hypertrophy and hyperplasia of type II pneumocytes and necrotic intra-alveolar debris associated with mild lymphocytic infiltration of the interstitium. HE. $\times 63$.

with bronchiolar necrosis, PCV2 immunoreactivity was very intense in macrophages, but absent in the bronchiolar epithelium.

Focal or multifocal PRRSV immunolabelling was demonstrated in the lungs of 19 animals $(67.8 \%)$ and this labelling ranged from slight to moderate in intensity. The signal was present in the cytoplasm of hypertrophic and hyperplastic pneumocytes and in the cytoplasm of some macrophages in the alveolar septa (Fig. 2b). ADV antigen was not demonstrated in any of the lungs and there was no labelling within negative control sections.
Overall, the lungs from eight animals $(28.6 \%)$ had PRRSV-PGV2 coinfection, while PGV2 monoinfection was present in the lungs of four animals $(14.3 \%)$ and PRRSV monoinfection in the lungs of 11 animals $(39.3 \%)$ (Table 1$)$. The lungs of five animals $(17.9 \%)$ were negative for both viruses. One of the coinfected lungs came from a pig that was seropositive for SIV and this animal came from a farm with confirmed PMWS.

The results of the present study therefore suggest that the major aetiological agents of PNP in Italian pigs are PRRSV and PGV2, as previously documented in Canadian (Drolet et al., 2003) and European (Pesch et al., 2000; Grau-Roma and Segalés, 2007) studies. However, the results of our investigation are more aligned with those of the Canadian study, in which PRRSV infection was more consistently demonstrated, rather than the European study that suggested a dominant role for PCV2 infection in PNP. This finding should be confirmed by further targeted sampling in animals with PNP.

In the present study, none of the three viral agents were detected in the lungs of five of 28 pigs $(17.9 \%)$ with histological changes consistent with PNP. This finding is not dissimilar to those of other investigations. Grau-Roma and Segalés (2007) detected no agents in eight out of 74 cases $(10.8 \%)$ and Drolet et al. (2003) described four out of 60 samples $(7 \%)$ as negative for all agents for which they tested. The relatively higher percentage of negative samples in the present study probably reflects the smaller number of agents
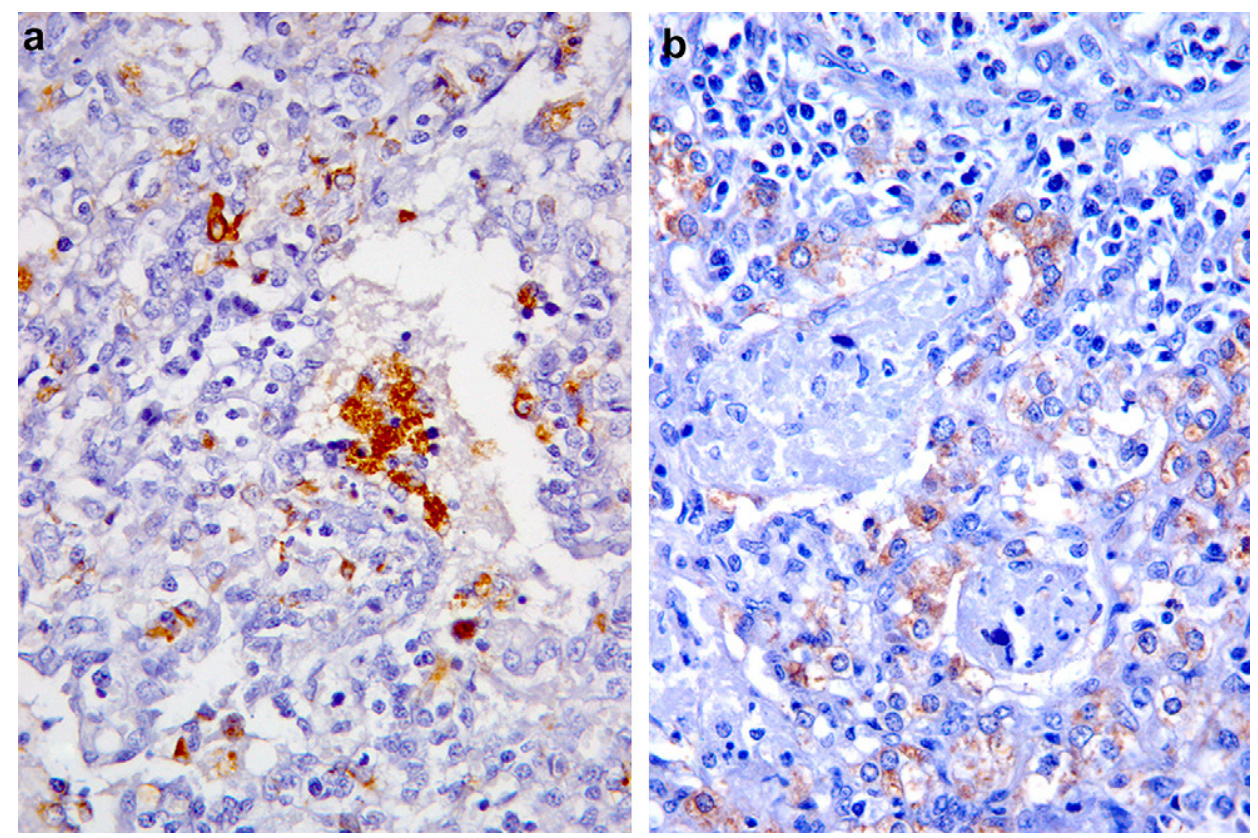

Fig. 2. (a) Intense labelling of PGV2 antigen within the cytoplasm of macrophages within the alveolar septa and in necrotic intra-alveolar material. (b) Weaker labelling of PRRSV antigen within the cytoplasm of type II pneumocytes. IHC. $\times 40$. 
Table 1

Summary of immunohistochemical labelling for PRRSV and PGV2

\begin{tabular}{lccc}
\hline & $\begin{array}{c}\text { PMWS + farms } \\
\text { PCV2 + lungs }\end{array}$ & $\begin{array}{c}\text { PMWS +farms } \\
\text { PCV2 - lungs }\end{array}$ & $\begin{array}{c}\text { PMWS-farms } \\
\text { PCV2 - lungs }\end{array}$ \\
\hline PRRSV + & $8^{*}$ & 5 & 6 \\
PRRSV - & 4 & 5 & 0 \\
\hline
\end{tabular}

PMWS, post-weaning multisystemic wasting syndrome; PCV2, porcine circovirus 2; PRRSV, porcine reproductive and respiratory syndrome virus.

*One animal was seropositive for swine influenza virus.

investigated. Additionally, the stage of infection at the time of sampling and the sensitivity of the immunohistochemical techniques used may impact upon successful identification of infectious agents.

The distribution of PCV2 and PRRSV antigens within the lungs reported here provides some insight into the pathogenesis of PNP. PRRSV antigen was mainly found in the cytoplasm of hypertrophic pneumocytes, while PCV2 antigen predominantly appeared in the cytoplasm of macrophages within the alveolar septa and alveolar space. PGV2 antigen was only associated with pneumocytes in the lungs of a single animal. These findings may suggest that PRRSV is associated with hypertrophy of pneumocytes, while PCV2 may be associated with necrosis. Hypertrophy and hyperplasia of type II pneumocytes are widely described in pulmonary disease of caesarean-derived, colostrum-deprived $(\mathrm{CD} / \mathrm{CD})$ piglets with experimental PRRSV infection (Halbur et al., 1996; Beyer et al., 1998; Harms et al., 2001). Necrotizing bronchiolitis and abundant necrotic debris in the alveolar spaces, associated with a high PGV2 load assessed by immunohistochemistry, were also reported by Drolet et al. (2003) and Grau-Roma and Segalés (2007). In contrast, experimental studies (Harms et al., 2001) report that coinfection by both viruses can cause more severe pulmonary disease than infections by a single virus.

In conclusion, the present study has confirmed that PNP in Italian pigs is more commonly associated with infection by PRRSV than with PCV2, although coinfection by both viruses may occur and appears to be related to increased disease severity.

\section{Acknowledgment}

The authors are grateful to A. Collins for English language editing of the text.

\section{References}

Austin R, Bystom J (1991) Proliferative and necrotizing pneumonia in Manitoba. Canadian Veterinary Journal, 32, 371 .
Beyer J, Fitchner D, Schirrmeier H, Granzow H, Polster U et al. (1998) Arterivirus PRRSV. Experimental studies on the pathogenesis of respiratory disease. Advances in Experimental Medicine and Biology, 440, 593-599.

Dea S, Bilodeau R, Sauvageau R, Montpetit C, Martineau GP (1992) Antigenic variant of swine influenza virus causing proliferative and necrotizing pneumonia in pigs. Journal of Veterinary Diagnostic Investigation, 4, 380-392.

Drolet R, Larochelle R, Morin M, Delisle B, Magar R (2003) Detection rates of porcine reproductive and respiratory syndrome virus, porcine circovirus type 2 , and swine influenza virus in porcine proliferative and necrotizing pneumonia. Veterinary Pathology, 40, 143-148.

Girard C, Morin R, Eialzhary Y (1992) Experimentally induced porcine proliferative and necrotizing pneumonia with an influenza A virus. Veterinary Record, 130, 206-207.

Grau-Roma L, Segalés J (2007) Detection of porcine reproductive and respiratory syndrome virus, porcine circovirus type 2, swine influenza virus and Aujeszky's disease virus in cases of porcine proliferative and necrotizing pneumonia (PNP) in Spain. Veterinary Microbiology, 119, 144-151.

Grieco V, Gelmetti D, Finazzi G, Brocchi E (1997) Immunohistologic diagnosis of pseudorabies (Aujeszky's disease) using monoclonal antibodies. Fournal of Veterinary Diagnostic Investigation, 9, 326-328.

Halbur PG, Paul PS, Meng XJ, Lum MA, Andrews JJ et al. (1996) Comparative pathogenicity of nine US porcine reproductive and respiratory syndrome virus (PRRSV) isolates in a five-week-old caesarean-derived, colostrum-deprived pig model. Fournal of Veterinary Diagnostic Investigation, $\mathbf{8}, 11-20$.

Harding JCS (2004) The clinical expression and emergence of porcine circovirus 2. Veterinary Microbiology, 98, 131-135.

Harms PA, Sorden SD, Halbur PG, Bolin SR, Lager KM (2001) Experimental reproduction of severe disease in $\mathrm{CD} / \mathrm{CD}$ pigs concurrently infected with type 2 porcine circovirus and porcine reproductive and respiratory syndrome virus. Veterinary Pathology, 38, 528-539.

Larochelle R, Sauvageau R, Magar R (1994) Immunohistochemical detection of swine influenza virus and porcine reproductive and respiratory syndrome virus in porcine proliferative and necrotizing pneumonia cases from Québec. Canadian Veterinary Journal, 35, 513-515.

Morin M, Girard C, Eiazhary Y, Fajardo R, Drolet R et al. (1990) Severe proliferative and necrotizing pneumonia in pigs: a newly recognized disease. Canadian Veterinary fournal, 31, 837-839.

Pesch S, Schmidt U, Ohlinger VF (2000) Proliferative and necrotizing pneumonia (PNP) is a result of co-infection with porcine reproductive and respiratory disease (PRRSV) and porcine circovirus type 2 (PCV2). Proceedings of the International Pig Veterinary Society Congress, 16, 581 .

Sarli G, Ostanello F, Morandi F, Fusaro L, Gnudi M et al. (2009) Application of a protocol for the diagnosis of postweaning multisystemic wasting syndrome in Italy. Veterinary Record, 164, 519-523. 
Segalés J, Balasci M, Domingo M, Carvalho LF, Pijoan C (1997) Immunohistochemical demonstration of the spread of pneumotropic strain 4892 of Aujezsky's disease virus in conventional pigs. Fournal of Comparative Pathology, 116, 387-395.

Segalés J, Rosell C, Domingo M (2004) Pathological findings associated with naturally acquired porcine circovi- rus type 2 associated disease. Veterinary Microbiology, 98, 137-149.

$\left[\begin{array}{l}\text { Received, fanuary 14th, } 2009 \\ \text { Accepted, fune 10th, } 2009\end{array}\right]$ 